\title{
Probability-based opportunity dynamic adaptation (PODA) of contention window for home M2M networks
}

\begin{abstract}
With the emergence of the Internet of Things (IoT), the growing use of autonomous sensing and actuating devices in areas such as smart grid, e-healthcare, home networking, and machine-tomachine (M2M) communication has become an important communication paradigm. Nonetheless, to fully exploit the applications facilitated by M2M communication, service requirements such as data throughput, scalability and reliability must be in place. This paper proposes a new backoff adaptation mechanism known as probability-based opportunity dynamic adaptation (PODA) for M2M communication using the IEEE 802.11ah protocol. The proposed PODA is an enhanced version of the binary exponential backoff (BEB) where a station estimates the number of contending stations in a distributed manner and adaptively tunes its minimum contention window (CW) prior to the contention process for better network throughput and packet delivery ratio. Owing to its great flexibility and ease of implementation, BEB has been extended to home M2M communication such as wireless sensor networks and smart grid technologies without relying on wide area communication. However, the current form of BEB has its shortcomings in the emerging M2M paradigm. The adaptation of $\mathrm{CW}$ in PODA is based on the optimal station's access opportunity to improve network performance instead of direct $\mathrm{CW}$ scaling. Using the proposed adaptation method, the network throughput can be improved by as much as 18 percent in the home M2M network studied, while enhancing network reliability and fairness.
\end{abstract}

Keyword: Dynamic adaptation; Performance improvement; Contention window; IEEE 802.11ah 